\title{
Beyond Procedural Facade Parsing: Bidirectional Alignment via Linear Programming
}

\author{
Mateusz Koziński, Guillaume Obozinski and Renaud Marlet \\ Université Paris-Est, LIGM (UMR CNRS 8049), ENPC \\ F-77455 Marne-la-Vallée
}

\begin{abstract}
We propose a novel formulation for parsing facade images with user-defined shape prior. Contrary to other state-of-the-art methods, we do not explore the procedural space of shapes derived from a grammar. Instead we formulate parsing as a linear binary program which we solve using dual decomposition. The algorithm produces plausible approximations of globally optimal segmentations without grammar sampling. It yields state-of-the-art performance on standard datasets.
\end{abstract}

\section{Introduction}

The goal of facade parsing is to segment rectified building images into regions corresponding to architectural elements, like windows, balconies and doors. The resulting segments have to satisfy structural constraints, e.g., alignment of windows on the same floor, or requirement that a balcony is associated to a window and right below it. Applications include creating 3D models of urban scenes.

A common approach to this problem is to let the user specify a shape prior encoding the structural constraints. It often takes the form of a shape grammar and proposed algorithms try to find a sequence of instantiated grammar rules yielding an optimal segmentation [1-3]. But the dimension of the search space is very large. Consequently, these algorithms suffer from the 'curse of structural exploration'. They search the solution space randomly [1,2], which does not guarantee optimality or repeatability, or severely subsample the image [3].

In this paper we lift the curse of structural exploration by proposing an altenative formulation of priors, which can be mapped into a linear binary program and solved efficiently, yielding state-of-the-art performance on standard datasets.

\subsection{Related Work}

Most proposed priors that are complex enough to model constraints of building facades rely on shape grammars [4]. The concept has been introduced by Stiny et al. [5] in the 70's, and the idea of representing image contents in a hierarchical and semantized manner traces back to the work of Ohta et al. [6,7]. Practical applications to image segmentation and interpretation are more recent [8-11].

A grammar is typically given by a set of nonterminal symbols $\mathcal{N}$, a set of terminal symbols $\mathcal{T}$, a start symbol in $\mathcal{N}$, and a set of production rules of the form $A_{0} \rightarrow A_{1} \ldots A_{n}$ where $A_{0} \in \mathcal{N}$ and $A_{i} \in \mathcal{N} \cup \mathcal{T}$ for $1 \leq i \leq n$. 
In the grammar of Han and Zhu [8], terminal symbols are rectangles and production rules combine them into rows, columns or grids, allowing rectangle nesting. The authors resort to a greedy algorithm for constructing the parse tree, which illustrates the difficulty of optimizing over a grammar derivation.

Drawing ideas from architectural modeling [12], where facade generation is analogous to string derivation in formal languages, the top-down parser of Teboul et al. [1,2] is one of the first attempts to parse facades using 'split grammars'. The input image is recursively split into rectangular subregions which are assigned a class label. Spliting directions as well as the number and class of subrectangles are non-deterministically chosen according to a predefined set of production rules. The process continues until all rectangles have a terminal class. The parser actually samples a number of possible derivations, only exploring a small part of the structural space. Even with a 'smart' sampling strategy [2], it does not produce repeatable results: as reported in [13], inference consists in independently running the exploration five times and keeping the best solution.

To counter the drawbacks of sampling, Riemenschneider et al. [3] propose an adaptation of the Cocke-Younger-Kasami (CYK) algorithm for parsing string grammars to two-dimensional split grammars. Its complexity is $O\left(w^{2} h^{2} N\right)$, where $w$ and $h$ are image dimensions and $N$ is the number of possible combinations of production rule attributes (including splitting positions). This limits practical applications of the algorithm to grids of about 60 by 60 cells. To circumvent this limitation the authors test different methods of image subsampling.

An attempt to fight the curse of procedural exploration was proposed by Koziński and Marlet [14], using graph grammars and MRF optimization. In contrast to parsers like [2] whose combinatorial search explores both the nature of splits and their position at the same time, sampling here concerns structure only; optimal positions for a given sampled structure are found with a principled and efficient method. The space to explore, which now does not depend on image size, is considerably smaller, but the curse of the procedural space is not eliminated completely as graph-grammar sampling remains.

Some facade segmentation methods $[15,16]$ do not use any user-defined shape prior. The bottom-up method proposed by Martinovic et al. [15] applies 'soft' architectural principles as a postprocessing step after image segmentation, but cannot accommodate 'hard' structural constraints. It can produce artifacts, like windows extending further than their balconies. A more recent work by Cohen et al. [16] uses a sequence of dynamic programs to recover a segmentation that respects a set of hard-coded constraints and attains state-of-the-art performance on the standard datasets. In our experiments, our method outperforms this algorithm while offering full flexibility with respect to shape prior specification.

In this paper we formulate the problem of finding an optimal segmentation as a binary linear program. We solve this program using the dual decomposition (DD) approach $[17,18]$. Similar techniques include Alternating Direction of Multipliers Method (ADMM) [19]. We chose DD because ADMM, although known to feature better convergence properties, requires solving quadratic subproblems. The experiments confirm that DD behaves well in our application. 
Table 1. Comparison of with state-of-the-art facade parsing methods.

\begin{tabular}{lccccc}
\hline Property & {$[2]$} & {$[15]$} & {$[16]$} & {$[3]$} & Ours \\
\hline User-defined shape prior & $\checkmark$ & - & - & $\checkmark$ & $\checkmark$ \\
Approximation of global optimum & - & - & $-*$ & $\checkmark$ & $\checkmark$ \\
No need of image subsampling (for tractability) & $\checkmark$ & $\checkmark$ & $\checkmark$ & - & $\checkmark$ \\
Simultaneous alignment in two dimensions & $\checkmark$ & $\checkmark$ & - & $\checkmark$ & $\checkmark$ \\
\hline
\end{tabular}

* Cohen et al. [16] can issue a certificate of optimality if the found solution is optimal.

\subsection{Contributions}

Our approach for image parsing does not suffer from the curse of procedural exploration. It is based on a shape prior formalism that allows efficient parsing.

Instead of expressing a shape prior using grammar rules, we propose to represent the structural decomposition of a scene as a hierarchy of classes, complemented by a specification of forbidden configurations of neighboring elements.

The parsing problem can then be turned into a linear binary program, which we solve efficiently using dual decomposition, eliminating the need for a procedural exploration of the solution space. As shown in the experiment section, our algorithm features the accuracy of methods using hard-coded structural constraints $[15,16]$ while retaining the flexibility of grammar-based methods $[2,3]$. The comparison to state of the art is summarized in table 1 .

\section{Proposed Model}

Although it departs from hte grammar-based approach, our structural segmentation framework is inspired by the process of hierarchical image subdivision into rectangular regions, which we will refer to as rectangles in the rest of the paper. The shape prior is encoded as a tree with nodes corresponding to classes of rectangles. Child nodes represent classes of rectangles resulting from splitting a rectangle of a parent class. We require that a rectangle of a class resulting from a vertical split can only be split horizontally and vice versa. This tree is complemented with a table of pairwise potentials associated to each nonterminal node. They can be used to penalize invalid or unlikely adjacency configurations of child rectangles. Our algorithm can handle infinite values of the potentials and in our experiments we only use binary potentials that take the value of zero or infinity, preventing some configurations of neighbors and allowing the others.

In contrast to split grammars, which are context-free and cannot be used to express simultaneous alignment in two dimensions (other than with implementation tricks that introduce some context dependency [2]), we require rectangles of the same class to be aligned both vertically and horizontally. This requirement can be enforced by constraining all rectangles of the same class that are aligned along the splitting direction to be split in the same positions into subrectangles of the same classes. A tree example and corresponding segmentations are presented in figure 1. Note the bidirectional alignment of windows (class $g$ ). 

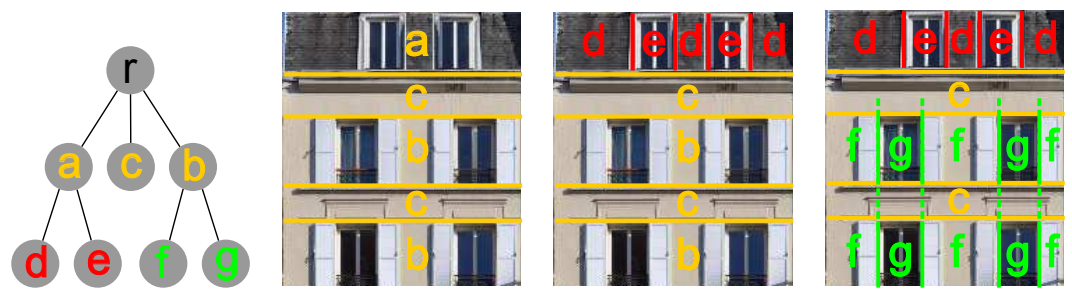

Fig. 1. A shape prior consists of a hierarchy of classes (image 1) and a table of pairwise potentials for each nonterminal node (not shown here). Each image (2-4) shows substitution of all rectangles of a particular class with rectangles of child classes.

\subsection{Optimal Segmentation as a Binary Linear Program}

We denote the set of indices of image pixels by $\mathcal{I}=\{(i, j) \mid i \in I, j \in J\}, I=$ $\{1, \ldots, h\}$ and $J=\{1, \ldots, w\}$, where $h$ is image height and $w$ is image width. We denote the set of rectangle classes by $\mathcal{C}=K \cup L$, where $K$ denotes the set of classes that result from a horizontal split, also called row-classes, and $L$ is the set of classes that result from a vertical split, called column-classes, and $K \cap L=\emptyset$. The root of the tree $r$ is a 'starting class', corresponding to the whole image. Without loss of generality we assume that $r$ is split horizontally and by convention we consider $r \in L$. We recall that nodes in $K$ can only have children in $L$ and vice versa. Consequently, all nodes at each level of the tree are either col-classes or row-classes. We denote the set of children of class $n \in \mathcal{C}$ by $C h(n)$ and the set of descendants of $n$, including $n$, by $\operatorname{Desc}(n)$. Similarly, we denote the set of ancestors of $n$, including $n$, by $A n c(n)$, and its parent by $P a(n)$. The set of siblings of $n$ is denoted $\operatorname{Sib}(n)$. We define a set of classes $t \in \mathcal{C}$ corresponding to the leaves of the tree by $T$ and call its members terminal classes.

A sequence of vertical and horizontal splits assigns a sequence of rectangle class labels to every pixel of the image. For any row $i$, it is thus possible to list all the classes that are assigned to at least one pixel on the row. Below we show that a segmentation consistent with a prior of the proposed form can be encoded in terms of the sets of classes assigned to each image row and column. This rowand column-based formulation enables global alignment of distant rectangles of the same class. We define variables $y_{i k}, y_{i l}, x_{j k}, x_{j l} \in\{0,1\}$ such that $y_{i k}=1$ if $k$ is present in row $i$ and $x_{j l}=1$ if $l$ appears in column $j$. We make a distinction between the variables encoding assignment of row-classes $k \in K$ and columnclasses $l \in L$, because they behave differently for horizontal and vertical splits.

In table 2 we present how the process of shape derivation changes the sets of row- and column-classes present in image rows and columns, and formulate constraints on $y_{i k}, y_{i l}, x_{j k}$ and $x_{j l}$ that reflect this behaviour. As shown in the second row of the table, a vertical split of a rectangle of parent class results in a number of rectangles of child classes. Because the split is along the vertical axis, only one child rectangle is going to appear in each image column previously occupied by the parent. However, all children are going to occur in each image row where the parent was present. We emphasize that all vertically aligned 
Table 2. Illustration of the splitting process and interpretation of the variables $x_{j l}$ and $y_{i k}$. The splitting process is just a concept that helps us to introduce our formulation and not a mode of operation of the proposed algorithm.

\begin{tabular}{|c|c|c|c|c|}
\hline \multirow{4}{*}{ 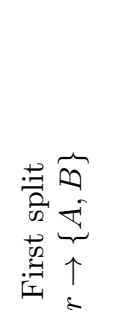 } & \multirow{2}{*}{\multicolumn{2}{|c|}{$\begin{array}{l}\text { Example } \\
\text { sequence } \\
\text { of splits } \\
\text { A }\end{array}$}} & $\begin{array}{l}\text { Specific constraints } \\
\text { for the example }\end{array}$ & \multirow{4}{*}{$\begin{array}{c}\forall k \in C h(r), x_{j k}=1 \\
\sum_{k \in C h(r)} y_{j k}=1\end{array}$} \\
\hline & & & \multirow{3}{*}{$\begin{array}{c}x_{j A}=1 \\
x_{j B}=1 \\
y_{i A}+y_{i B}=1\end{array}$} & \\
\hline & & $B$ & & \\
\hline & \multicolumn{2}{|c|}{$A$} & & \\
\hline \multirow{3}{*}{ 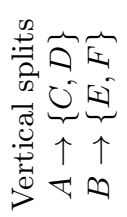 } & $\mathrm{C}$ & $\mathrm{D}$ & \multirow{3}{*}{$\begin{array}{c}x_{j C}+x_{j D}=x_{j A} \\
x_{j E}+x_{j F}=x_{j B} \\
y_{i A}=y_{i C}=y_{i D} \\
y_{i B}=y_{i E}=y_{i F}\end{array}$} & \multirow{3}{*}{$\begin{array}{c}\forall k \in K, \sum_{l \in C h(k)} x_{j l}=x_{j k} \\
\forall k \in K, \forall l \in C h(k), y_{j l}=y_{j k}\end{array}$} \\
\hline & \multicolumn{2}{|c|}{\begin{tabular}{l|l}
$\mathrm{E}$ & $\mathrm{F}$ \\
\end{tabular}} & & \\
\hline & $\mathrm{C}$ & $D$ & & \\
\hline \multirow{3}{*}{ 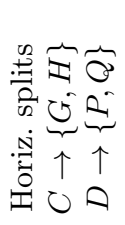 } & \begin{tabular}{l|l}
$\mathrm{G}$ \\
$\mathrm{H}$
\end{tabular} & $\begin{array}{l}P \\
Q\end{array}$ & \multirow{3}{*}{$\begin{aligned} x_{j C} & =x_{j G}=x_{j H} \\
x_{j D} & =x_{j P}=x_{j Q} \\
y_{i C} & =y_{i G}+y_{i H} \\
y_{i D} & =y_{i P}+y_{i Q}\end{aligned}$} & \multirow{3}{*}{$\begin{array}{l}\forall l \in L, \forall k \in C h(l), x_{j k}=x_{j l} \\
\quad \forall l \in L, \sum_{k \in C h(l)} y_{i k}=y_{i l}\end{array}$} \\
\hline & $E$ & $\mathrm{~F}$ & & \\
\hline & $\mathrm{H}$ & $P$ & & \\
\hline
\end{tabular}

rectangles of the same class are split simultaneously along the same lines, so that the child rectangles are aligned and their classes are consistent along the splitting axis. The same reasoning applies to horizontal splits.

The corresponding constraints on $x_{j l}, x_{j k}, y_{i l}$ and $y_{i k}$ are presented in the third column of table 2 . We note that for vertical splits the state of each $y_{i l}$ for $l \in C h(k)$ is determined by $y_{i k}$ and that the same holds for horizontal splits, $x_{j k}$ and $x_{j l}$, as shown in the fourth column of table 2 . We eliminate $x_{j k}$ and $y_{i l}$ by combining the two first equations and the two second equations from rows two and three of the table. We get

$$
\forall i \in I, \forall l \in \stackrel{\circ}{L}, \sum_{k^{\prime} \in C h(l)} y_{i k^{\prime}}=y_{i P a(l)}, \quad \forall j \in J, \forall k \in \stackrel{\circ}{K}, \sum_{l^{\prime} \in C h(k)} x_{j l^{\prime}}=x_{j P a(k)},
$$

where $\stackrel{\circ}{L}=L \backslash(T \cup\{r\})$ and $\stackrel{\circ}{K}=K \backslash T$. We visualize the domain of the constraints in fig. 2.

In the interest of maintaining the convention of assigning row-classes $k \in K$ to rows and column-classes $l \in L$ to columns, we modify the constraint from the first row of the table. We require that the root class is assigned to each column 


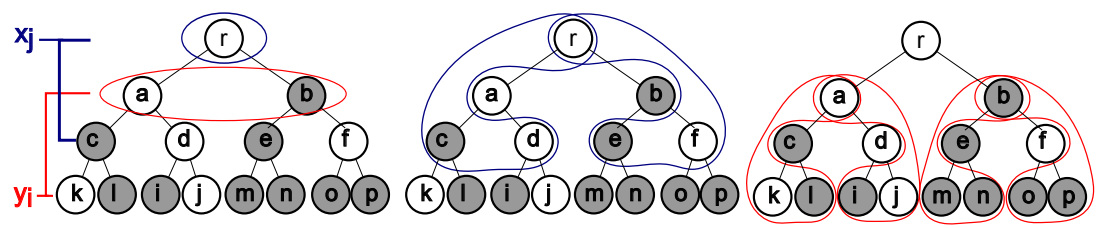

Fig. 2. Visualization of the state of variables $y_{i k}$ and $x_{j l}$ for some pixel $i, j$. The white nodes correspond to classes $k$ and $l$ for which $y_{i k}=1$ and $x_{j l}=1$. The gray nodes correspond to classes with $y_{i k}=0$ or $x_{j l}=0$. The domains of constraints (1) on $y_{i k}$ are circled in blue and the domains of constraints on $x_{j l}$ are circled in red. Left: the domains of (1b). Middle and right: the domains of (1a). Note that only one leaf is connected to the root by a path of white nodes. This illustrates the uniqueness of pixel class given the state of variables corresponding to its row and column.

and that the first horizontal split assigns a unique class to each row:

$$
\forall j \in J, x_{j r}=1, \quad \forall i \in I, \sum_{k \in C h(r)} y_{i k}=1 .
$$

A key, albeit nontrivial consequence of constraints (1) is that there is a unique class assigned to each pixel at a certain level of the tree, which is unambiguously identified by the sets of classes present in the corresponding row and column.

Lemma 1. Consider a hierarchy of classes given as a tree, as defined earlier. Denote the depth of the tree by $M$, the set of column-classes at the $m$-th level of the tree by $L^{m}$ and the set of row-classes at the $m$-th level of the tree by $K^{m}$. Note that $L^{m}$ is nonempty only for even $m$ and $K^{m}$ for odd $m$. Denote the vectors of $y_{i k}$ and $x_{j l}$ by $\mathbf{y}$ and $\mathbf{x}$. Denote the set of $\mathbf{y}$ and $\mathbf{x}$ satisfying the constraint (1), enforcing the hierarchical structure of row-class and column-class assignment, by $C_{h}$. Then

$$
\begin{aligned}
(\mathbf{y}, \mathbf{x}) \in C_{h} \Longrightarrow & \forall(i, j) \in \mathcal{I} \quad \forall m \in\{0, \ldots, M\}, \\
\exists ! l_{i j}^{m} \in L^{m}: & \forall n \in A n c\left(l_{i j}^{m}\right), \quad\left(x_{j n}=1\right) \vee\left(y_{i n}=1\right) \quad \text { if } m \text { is even } \\
\exists ! k_{i j}^{m} \in K^{m}: & \forall n \in A n c\left(k_{i j}^{m}\right), \quad\left(x_{j n}=1\right) \vee\left(y_{i n}=1\right) \quad \text { if } m \text { is odd } .
\end{aligned}
$$

In words, for any pixel $(i, j) \in \mathcal{I}$, for any values of variables $y_{i k}$ and $x_{j l}$, that satisfy constraints (1), at any depth of the tree there exists exactly one rowclass, or one column-class such that the variables $x_{j l}$ and $y_{i k}$ corresponding to the class and all its ancestors are equal to one.

Proof. We prove the lemma by induction on the depth of the tree.

The root $r$ is the only node at depth $m=0$ of the tree and, by constraint (1b), it holds that $x_{j r}=1$ for all $j \in J$. Therefore the lemma holds for $m=0$.

For depth $m=1$ the tree is formed of the root and its children. By constraints (1b), we have that for each $i$ there exists a single $k_{i} \in C h(r)$ such that $y_{i k_{i}}=1$ and $y_{i k}=0$ for $k \neq k_{i}$. This proves the lemma for the case of a tree of depth 1 . 
Assume lemma 1 holds at depth $m$. If the level is of class $l \in L$, then by assumption for each $i$ we have a single $l_{j}^{m}$ such that the variables associated to all its ancestors are equal one. By constraint (1a), exactly one child of $l_{j}^{m}$ will have its associated variable $y_{i k_{i}^{m}}$ equal to one. Similar reasoning applies if the level is of row-class type.

We model the assignment of terminal classes to pixels by variables $z_{i j t} \in$ $\{0,1\}$, where $z_{i j t}=1$ if pixel $(i, j)$ is of class $t \in T$ and $z_{i j t}=0$ otherwise. A single terminal class has to be assigned to each pixel

$$
\forall(i, j) \in \mathcal{I}, \quad \sum_{t \in T} z_{i j t}=1 .
$$

By lemma 1, all ancestors of the class assigned to pixel $(i, j)$ have the variables $y_{i k}$ and $x_{j l}$ equal to one, which leads to the inequalities

$$
\forall(i, j) \in \mathcal{I}, \forall k \in K, \sum_{t \in \operatorname{Desc}(k)} z_{i j t} \leq y_{i k}, \quad \forall(i, j) \in \mathcal{I}, \forall l \in L, \sum_{t \in \operatorname{Desc}(l)} z_{i j t} \leq x_{j l} .
$$

Each nonterminal class has a table of pairwise potentials defined on its children. The potentials determine the likelihood of observing neighboring rectangles of the child classes. We implement the potentials with variables $y_{i k k^{\prime}}$ and $x_{j l l^{\prime}}$

$$
\begin{aligned}
& \forall i \in\{1, \ldots, h-1\}, \forall k \in K \sum_{k^{\prime} \in \operatorname{Sib}(k)} y_{i k k^{\prime}}=y_{i k}, \\
& \forall i \in\{1, \ldots, h-1\}, \forall k^{\prime} \in K \sum_{k \in \operatorname{Sib}\left(k^{\prime}\right)} y_{i k k^{\prime}}=y_{i+1 k^{\prime}}, \\
& \forall j \in\{1, \ldots, w-1\}, \forall l \in L \sum_{l^{\prime} \in S i b(l)} x_{j l l^{\prime}}=x_{j l}, \\
& \forall j \in\{1, \ldots, w-1\}, \forall l^{\prime} \in L \sum_{l \in \operatorname{Sib}\left(l^{\prime}\right)} x_{j l l^{\prime}}=x_{j+1 l^{\prime}} .
\end{aligned}
$$

We denote the cost of assigning type $t$ to pixel $(i, j)$ by $c_{i j t}$, and the pairwise cost for column- and row-classes by $c_{k k^{\prime}}$ and $c_{l l^{\prime}}$. We define the sets of pairs of row- and column-classes that are siblings in the tree by $S K$ and $S L$. The segmentation task can be formulated as minimizing the following objective

$$
E=\sum_{(i, j) \in \mathcal{I}} \sum_{t \in T} z_{i j t} c_{i j t}+\sum_{i=1}^{h-1} \sum_{\left(k, k^{\prime}\right) \in S K} y_{i k k^{\prime}} c_{k k^{\prime}}+\sum_{j=1}^{w-1} \sum_{\left(l, l^{\prime}\right) \in S L} x_{j l l^{\prime}} c_{l l^{\prime}}
$$

subject to constraints (4) to (6).

\section{Inference}

The formulated problem is linear and has a large number of binary variables. We relax the binary domain constraint and let the variables take values within the range $[0,1]$. We apply dual decomposition to the resulting continuous problem. 


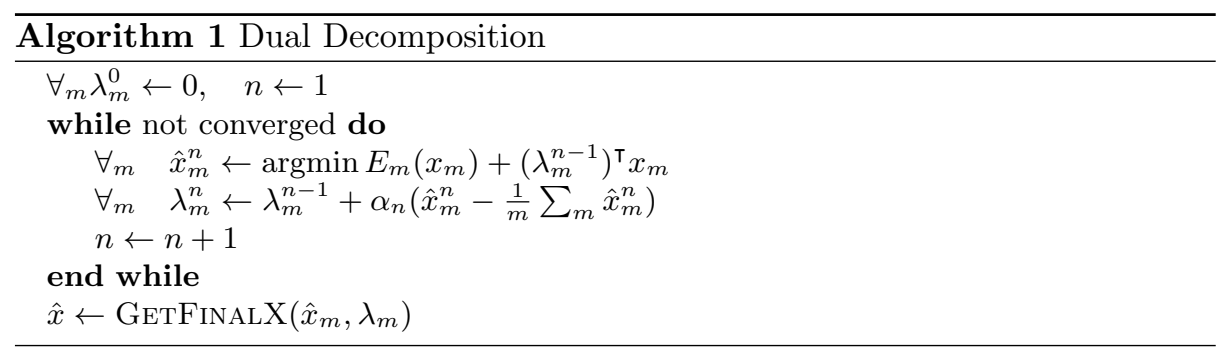

\subsection{The Dual Decomposition Algorithm}

The dual decomposition algorithm is based on the idea of decomposing a difficult problem into a number of 'slave' subproblems that are easy to solve. Given an original problem $\hat{x}=\operatorname{argmin} \sum_{m} E_{m}(x), x \in C$, where $C$ denotes the feasible set, we construct a number of copies of the variable $x$, denoted $x_{m}$, and couple them by means of a new constraint $x_{m}=x$. We formulate the dual problem $\max _{\lambda_{m}} \min _{x, x_{m}} \sum_{m}\left(E_{m}\left(x_{m}\right)+\lambda_{m}^{\top}\left(x-x_{m}\right)\right)$, subject to $x_{m} \in C$, where $\lambda_{m}$ is a vector of Lagrange multipliers. The problem is solved using a projected subgradient algorithm. Calculating subgradient of the dual objective requires solving $\hat{x}_{m}=\operatorname{argmin} E_{m}\left(x_{m}\right)+\lambda_{m}^{\top} x_{m}$, subject to $x_{m} \in C$, separately for each $m$. The latter minimizations are called slave problems. We refer the reader to $[17,18]$ for the derivation. We denote the values of variables in iteration $n$ by a superscript and $\alpha$ denotes the step size. The algorithm is run with decaying step size. The values of $\hat{x}_{m}$ eventually converge and heuristics, represented in algorithm 1 by procedure GETFinalX, can be used to decide on the components of $\hat{x}$ on which $\hat{x}_{m}$ disagree [17].

The main design decision to be made when applying dual decomposition is how to decompose the original objective function into slave objectives. The main criterion is the ability to efficiently solve the slave problems. Below we present a decomposition of the objective (7) into subproblems that can be solved by means of dynamic programming in time linear in the number of pixels.

\subsection{Application of Dual Decomposition to the Problem}

To make the slave problem tractable we need to decouple the variables $y_{i n}$ corresponding to image rows from the variables $x_{j n}$ corresponding to columns. This results in slaves assigning sets of classes to rows or columns and terminal classes to pixels.

This decoupling is however not sufficient since feasible configurations of the sets, encoded by vectors $\left(y_{i k}\right)$ and $\left(x_{j l}\right)$, are determined by constraints $(1)$, that have a complex structure. We propose a further decomposition, that results in a larger number of slaves with simpler constraints. The slaves assign a single class to each pixel and each image row or column.

Each instantiation of constraints (1) can be transformed by recursively plugging its left-hand side to a left-hand side of another equation of type (1) until 


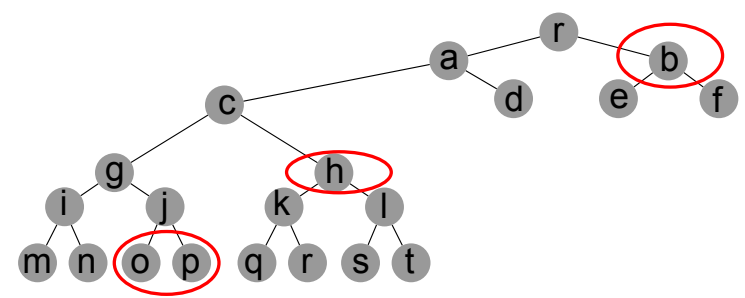

Fig. 3. The structure of set $H_{l}$, for $l=j$, visualized on a tree of classes. Elements of the set are outlined in red. Exactly one of the elements has to be assigned to a single image row.

the resulting sum equals one. Consequently, we get

$$
\forall l \in L \backslash T \quad \sum_{k \in H_{l}} y_{i k}=1, \quad \forall k \in K \backslash T \quad \sum_{l \in V_{k}} x_{j l}=1
$$

The sets $V_{k}$ and $H_{l}$ reflect the substitution process described above. The set $V_{k}$ can be described as the smallest set containing all children of $k$ and such that if some $l$ belongs to $V_{k}$, then all the siblings of its grandparent do as well. Formally, $V_{k}=C h(k) \cup\left[L \cap\left(C h\left(A_{k}\right) \backslash A_{k}\right)\right]$, where $A_{k}=A n c(k)$ and by $C h\left(A_{k}\right)$ we denote the set of all children of all elements of $A_{k}$. The structure of the set is illustrated in figure 3 . The set $V_{k}$ is defined in a similar way. Note that it is possible to transform (8) back to (1). It is enough to subtract from constraint (8) for some $l \in L \backslash T$ a constraint of the same type for $l^{\prime}=P a(P a(l))$ to get a constraint of type (1). The reader can verify that on the example presented in figure 3 . Thus, constraints (8) are equivalent to their original form (1).

The advantage of constraint (8) is that it is an intersection of simplex constraints, which entails that the problem can be naturally decomposed into a number of subproblems, one for each $l \in L \backslash T$ and each $k \in K \backslash T$.

\subsection{Structure of Slave Subproblem}

We thus create one slave for each $l \in L \backslash T$ and one for each $k \in K \backslash T$. Below we present the structure of a slave subproblem for some $l$. The slaves for $k$ are created symmetrically. We denote by $S H_{l}$ the set of pairs of sibling row classes $k, k^{\prime}$ such that $k, k^{\prime} \in H_{l}$. The copies of the variables are denoted with superscripts $l$. New cost coefficients $\tilde{c}_{i j t}=\frac{c_{i j t}}{(|L \backslash T|+|K \backslash T|)}$ and $\tilde{c}_{k k^{\prime}}=\frac{c_{k k^{\prime}}}{n_{k k^{\prime}}}$ are introduced, where $n_{k k^{\prime}}$ is the number of times the pair $k, k^{\prime}$ appears in different slaves. The objective is

$$
\min _{z_{i j t}^{l}, y_{i k}^{l}, y_{k k^{\prime}}^{l}} \sum_{\substack{i, j) \in \mathcal{I} \\ t \in T}}\left(\tilde{c}_{i j t}+\lambda_{i j t}^{l}\right) z_{i j t}^{l}+\sum_{\substack{i \in I \\ k \in H_{l}}} \lambda_{i k}^{l} y_{i k}^{l}+\sum_{\substack{i \in\{1, \ldots, h-1\} \\ k, k^{\prime} \in S H_{l}}} \tilde{c}_{k k^{\prime}} y_{i k k^{\prime}}^{l},
$$


where $\lambda_{i j t}^{l}$ is a Lagrange multiplier corresponding to a constraint coupling the variables $z_{i j t}^{l}$ for different slaves and $\lambda_{i k}^{l}$ is a Lagrange multiplier coupling the variables $y_{i k}^{l}$ for different slaves. The derivation of the slave objective from the original objective (7) is straightforward and is omitted here but detailed in the supplementary material. The feasible set of each slave problem is a projection of the original feasible set, defined by constraints (4) to (6), to the space of the slave variables:

$$
\begin{gathered}
\forall(i, j) \in \mathcal{I}, \quad \forall t \in T, \quad z_{i j t}^{l} \geq 0, \quad \forall(i, j) \in \mathcal{I}, \quad \sum_{t \in T} z_{i j t}^{l}=1, \\
\forall i \in I, \quad \forall k \in H_{l}, \quad y_{i k}^{l} \geq 0, \quad \forall i \in I, \quad \sum_{k \in H_{l}} y_{i k}^{l}=1, \\
\forall(i, j) \in \mathcal{I}, \quad \forall k \in H_{l} \quad \sum_{t \in \operatorname{Desc}(k)} z_{i j t}^{l} \leq y_{i k}^{l}, \\
\forall i \in J \backslash\{h\}, \quad \forall k \in H_{l}, \quad \sum_{k^{\prime} \in S i b^{l}(k)} y_{i k k^{\prime}}^{l}=y_{i k}^{l}, \\
\forall i \in J \backslash\{h\}, \quad \forall k^{\prime} \in H_{l}, \quad \sum_{k \in S i b^{l}\left(k^{\prime}\right)} y_{i k k^{\prime}}^{l}=y_{i+1 k^{\prime}}^{l},
\end{gathered}
$$

where $\operatorname{Sib}^{l}(k)$ denotes the set of sibling of class $k$ that belong to the set $H_{l}$. The nonnegativity constraints (10a) and (10b) are introduced due to the relaxation of the variables from binary to continuous domain. Constraints (10c) to (10e) have the same form as their corresponding ones in the original problem (4) to (6). The constraint (10b) on the sum of $y_{i k}^{l}$ represents constraints (1) and (1b) of the original problem, transformed according to (8). Summarizing, an intersection of the feasible sets of the slaves is equivalent to the feasible set of the original problem, in the sense that if for all $i, j, k, l, t$ we have $z_{i j t}=z_{i j t}^{k}=z_{i j t}^{l}$ and $y_{i k}=y_{i k}^{l}, x_{j l}=x_{j l}^{k}$, then $(z, y, x) \in C \quad \Longleftrightarrow \quad \forall l \in L \backslash T,\left(z^{l}, y^{l}\right) \in C^{l} \wedge$ $\forall k \in K \backslash T,\left(z^{k}, x^{k}\right) \in C^{k}$, where $C, C^{k}$ and $C^{l}$ denote the feasible sets of the original problem and of the slaves, respectively.

\subsection{Solving the Slave Subproblem}

It can be proven that the linear problem of each slave has integral vertices. In consequence, each slave can be seen as a labelling problem where we assign a label $k \in H_{l}$ to each row $i$ and a label $t$ to each pixel $(i, j) \in \mathcal{I}$. We find the optimal labelling by means of a dynamic program.

Given row class $k$ assigned to row $i$ by slave $l$, it is easy to determine the optimal classes $t_{i j}^{l k}$ for all pixels in the row. Constraint (10c) restricts the set of pixel classes that can be used in the row to ones that are descendants of $k$ or to ones that are not descendants of any $k \in H_{l}$, denoted $\tilde{T}_{l}=T \backslash \bigcup_{k \in H_{l}} \operatorname{Desc}(k)$. The optimal index is

$$
t_{i j}^{l k}=\underset{t \in \operatorname{Desc}(k) \cup \tilde{T}_{l}}{\operatorname{argmin}}\left(\tilde{c}_{i j t}+\lambda_{i j t}^{l}\right) .
$$




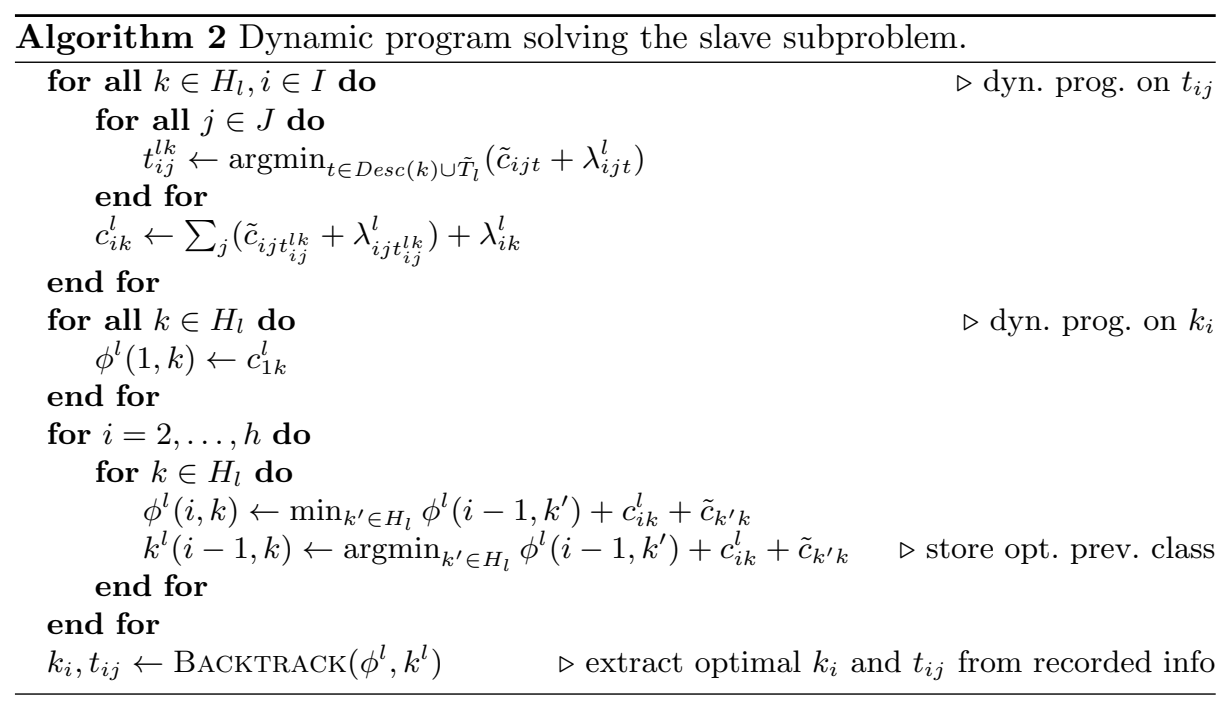

From objective (9) we derive the optimal cost of assigning row class $k$ to image row $i$, which is the sum of costs for each pixel and the per-row cost

$$
c_{i k}^{l}=\sum_{j=1}^{w}\left(\tilde{c}_{i j t_{i j}^{l k}}+\lambda_{i j t_{i j}^{l k}}^{l}\right)+\lambda_{i k}^{l}
$$

The optimal cost of assigning classes for the $i$ first rows, denoted $\phi^{l}(i, k)$, where $k$ is the row class assigned to row $i$, can be recursively defined as

$$
\phi^{l}(i, k)= \begin{cases}c_{1 k}^{l} & \text { if } i=1 \\ \min _{k^{\prime} \in H_{l}} \phi^{l}\left(i-1, k^{\prime}\right)+c_{i k}^{l}+\tilde{c}_{k^{\prime} k} & \text { otherwise. }\end{cases}
$$

We use this recursive structure of the subproblem to formulate the Viterbi algorithm 2 for finding its optimal solution. In the first step the optimal pixel indices $t_{i j}^{l k}$ are determined for each pixel and each $k \in H_{l}$ according to (11). They are then used for determining row class $\operatorname{costs} c_{i k}^{l}$ according to (12). Finally the Viterbi recursion of equation (13) is used and the optimal row and pixel classes are retrieved by backtracking.

\section{Experiments}

We tested the performance of our algorithm on facade parsing with two datasets. For each of them, we have created a shape prior consisting of a tree hierarchy of classes and, for each nonterminal node, a table of pairwise potentials. We use very simple, binary potentials, which penalize invalid ordering of rectangle classes, like sky under wall, with infinite cost. For each image, we run the DD algorithm for 200 iterations, with a fixed step-size sequence $\alpha_{n}=a / \sqrt{n}$, where $n$ 
Table 3. Performance on the ECP dataset. The rows corresponding to classes present class accuracy (the diagonal entries of confusion matrices, or recall). The bottom rows contain average class accuracy and total pixel accuracy. Starting from left, we present the performance of three layers of Martinovic's solution [15], and the results of Cohen et al. [16], using 'raw' per-pixel energies, and with SVM scores on top of the energies.

\begin{tabular}{|l|c|c|c|c|c|c|c||ccccccc|c|}
\multicolumn{1}{c|}{} & {$[15]-\mathrm{L} 1$} & {$[15]-\mathrm{L} 2$} & {$[15]-\mathrm{L} 3$} & {$[16]$} & {$[16]-S V M$} & Ours & Our confusion matrix & \\
\hline roof & 70 & 73 & 74 & 93 & 90 & 91 & 91 & 0 & 0 & 2 & 2 & 0 & 5 & roof \\
shop & 79 & 86 & 93 & 96 & 94 & 95 & 0 & 95 & 0 & 0 & 0 & 0 & 4 & shop \\
balcony & 74 & 71 & 70 & 92 & 91 & 90 & 1 & 0 & 90 & 0 & 4 & 0 & 5 & balc. \\
sky & 91 & 91 & 97 & 96 & 97 & 96 & 4 & 0 & 0 & 96 & 0 & 0 & 0 & sky \\
window & 62 & 69 & 75 & 87 & 85 & 85 & 3 & 1 & 4 & 0 & 85 & 0 & 5 & wind. \\
door & 43 & 60 & 67 & 82 & 79 & 74 & 0 & 22 & 0 & 0 & 0 & 74 & 4 & door \\
wall & 92 & 93 & 88 & 88 & 90 & 91 & 1 & 3 & 2 & 0 & 3 & 0 & 91 & wall \\
\hline class aver. & 73.0 & 77.6 & 80.6 & 90.6 & 89.4 & 88.8 & & & & & & & \\
pixel accur. & 82.6 & 85.1 & 84.2 & 90.3 & 90.8 & $\mathbf{9 2 . 3}$ & & & & & & & \\
\hline
\end{tabular}

is the number of iterations and $a$ is a constant. In our experiments the algorithm run for less than 4 minutes per a single image.

The ECP dataset [2] consists of about 100 rectified images of Haussmannian building facades with annotations segmenting the images into 7 classes: sky, roof, wall, window, balcony, shop and door. An issue is that this ground-truth annotation was created to be consistent with possible derivations of the grammar in [2], even when it cannot accomodate observations. For instance, attic windows are systematically aligned with facade windows, even when actually offset, and balconies can only extend throughout the entire facade or be constrained to a single window cavities, which is incorrect on a number of images. For this reason, we use the ground truth provided by [15]. It evades any structural interpretation: balconies and windows can be slightly misaligned, small pieces of doors may float above the ground, etc. But it is more accurate in terms of pixel classification.

The Graz50 dataset [3] is composed of 50 rectified images of building facades of different architectural styles. The facades feature more structural variation than the ones of the ECP dataset. The set of labels includes four classes: sky, wall, window and door.

Performance on the ECP Dataset has been tested using per-pixel energies that follow the description from [16]. We use a multi-feature extension of TextonBoost, as implemented by the authors of [20]. We use SIFT and Color SIFT descriptors, Local Binary Patterns and location features. The features of each type are clustered using K-means into 512 clusters. We establish a neighbourhood of 200 random rectangles and the final feature vector is a concatenation of histograms of cluster members' appearance in these rectangles. The per-pixel costs $c_{i j t}$ result from multi-class boosting [21]. We follow the protocol of [15] and [16] in performing the experiments on five folds with 80 training images and 20 testing images. The results are presented in table 3. Our method outperforms 
Table 4. Results of experiment on the Graz50 dataset. The second and third columns of the table show diagonal entries of the confusion matrices for results reported by Riemenschneider et al. [3] and our results. The right-hand side of the table contains the confusion matrix for our results. An example result is shown on the right hand side.

\begin{tabular}{|l|c|c||cccc|l|}
\cline { 2 - 5 } \multicolumn{1}{c|}{} & {$[3]$} & Ours & \multicolumn{3}{c|}{ conf. mat. } & \multicolumn{1}{|c|}{} \\
\hline sky & 91 & 93 & 93 & 0 & 0 & 6 & sky \\
window & 60 & 82 & 0 & 82 & 0 & 17 & window \\
door & 41 & 50 & 0 & 14 & 50 & 36 & door \\
wall & 84 & 96 & 0 & 3 & 0 & 96 & wall \\
\hline class average & 69.0 & 80.3 & & & & \\
total pixel accur. & 78.0 & $\mathbf{9 1 . 8}$ & & & & & \\
\hline
\end{tabular}

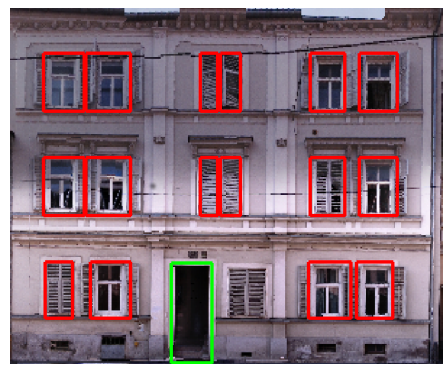

the one presented in [16] by a small margin. The main advantage of our algorithm is that it can accept any user-defined shape prior on input, which makes it more general. This shape prior can express constraints on the alignment of architectural elements in two dimensions, which is beyond the expressive power of the dynamic program proposed in [16].

Performance on the Graz50 dataset has been tested using the same type of pixel costs as for the ECP dataset. Five folds were used, splitting each time the dataset into 40 training images and 10 test images. The results are presented in table 4. One reason why our results are superior to those in [3] is that their method requires severe subsampling of the image to be tractable. Our method is more computationally efficient and can be run on full-resolution images.

\section{Conclusion and Future Work}

We have presented a novel approach to grammar-based facade analysis in which the task of parsing is formulated as an integer program. Our formulation does not suffer from the curse of procedural exploration, that is typical for existing split grammar parsers. It enables approximating globally optimal segmentations by means of state-of-the-art optimization algorithms. We have established new state-of-the-art level of performance on the ECP and Graz50 facade datasets.

As a direct extension of this work, we are considering learning the pairwise potentials using the approach presented in [22], which uses Dual Decomposition and that can accomodate the slaves defined in this paper (with modified costs). As increasingly good results have recently been observed on the problem of parsing rectified facade images, we see the relevance of addressing difficulties commonly appearing in real life images. These include modeling three-dimensional geometry of buildings and their projection on the image plane, as well as handling occlusions (e.g., by cars, vegetation, pedestrians or other buildings). 

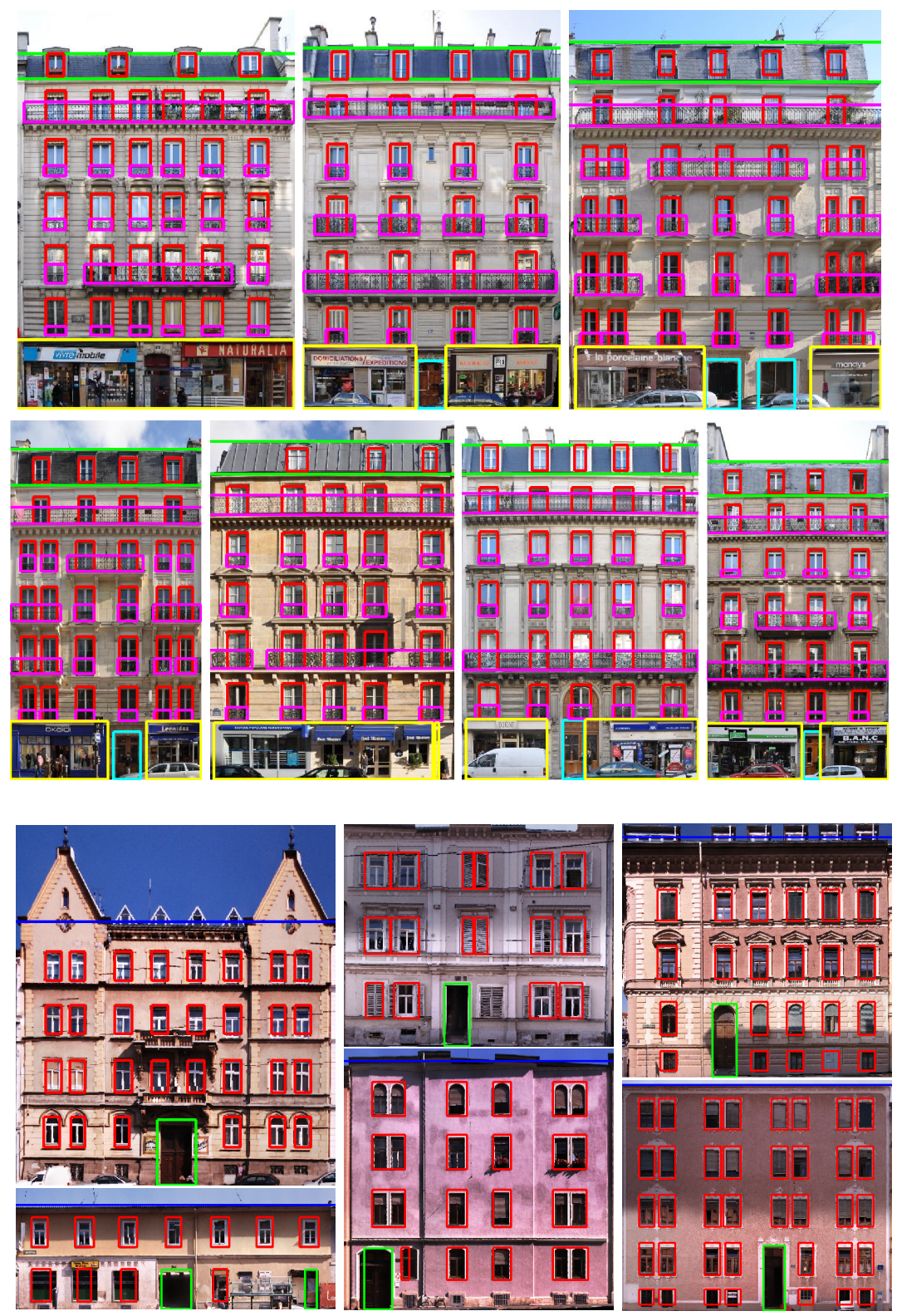

Fig. 4. Example parsing results on the ECP dataset (top) and on the Graz50 dataset (bottom). Green lines separate sky from roof and roof from facade. Balconies are outlined in magenta, shops in yellow, and doors in cyan. Note the variety of alignment patterns supported by the algorithm (top right). Typical errors are missed doors and missed roof windows. 
Acknowledgements This work was carried out in IMAGINE, a joint research project between Ecole des Ponts ParisTech (ENPC) and the Scientific and Technical Centre for Building (CSTB). It was partly supported by ANR project Semapolis ANR-13-CORD-0003.

\section{References}

1. Teboul, O., Simon, L., Koutsourakis, P., Paragios, N.: Segmentation of building facades using procedural shape priors. In: CVPR. (2010) 3105-3112

2. Teboul, O., Kokkinos, I., Simon, L., Koutsourakis, P., Paragios, N.: Shape grammar parsing via reinforcement learning. In: CVPR. (2011) 2273-2280

3. Riemenschneider, H., Krispel, U., Thaller, W., Donoser, M., Havemann, S., Fellner, D., Bischof, H.: Irregular lattices for complex shape grammar facade parsing. In: CVPR. (2012)

4. Zhu, S.C., Mumford, D.: A stochastic grammar of images. Foundations and Trends in Computer Graphics and Visions 2 (2006) 259-362

5. Stiny, G.N.: Pictorial and Formal Aspects of Shape and Shape Grammars and Aesthetic Systems. PhD thesis, University of California, Los Angeles (1975) AAI7526993.

6. Ohta, Y., Kanade, T., Sakai, T.: An analysis system for scenes containing objects with substructures. In: Proceedings of the Fourth International Joint Conference on Pattern Recognitions. (1978) 752-754

7. Ohta, Y., Kanade, T., Sakai, T.: A production system for region analysis. In: Proceedings of the Sixth International Joint Conference on Artificial Intelligence. (1979) 684-686

8. Han, F., Zhu, S.C.: Bottom-up/top-down image parsing with attribute graph grammar. IEEE Transactions on Pattern Analysis and Machine Intelligence 31 (2009) 59-73

9. Wang, W., Pollak, I., Wong, T.S., Bouman, C.A., Harper, M.P.: Hierarchical stochastic image grammars for classification and segmentation. IEEE Transactions on Image Processing 15 (2006) 3033-3052

10. Jin, Y., Geman, S.: Context and hierarchy in a probabilistic image model. In: CVPR (2). (2006) 2145-2152

11. Ahuja, N., Todorovic, S.: Connected Segmentation Tree - A Joint Representation of Region Layout and Hierarchy. In: CVPR. (2008)

12. Müller, P., Wonka, P., Haegler, S., Ulmer, A., Van Gool, L.: Procedural modeling of buildings. ACM Transations on Graphics 25 (2006) 614-623

13. Teboul, O.: Shape Grammar Parsing : Application to Image-based Modeling. PhD thesis, Ecole centrale Paris (2011)

14. Koziński, M., Marlet, R.: Image parsing with graph grammars and markov random fields. In: WACV. (2014)

15. Martinovic, A., Mathias, M., Weissenberg, J., Van Gool, L.: A three-layered approach to facade parsing. In: ECCV, Springer (2012)

16. Cohen, A., Schwing, A., Pollefeys, M.: Efficient structured parsing of facades using dynamic programming. In: CVPR. (2014)

17. Komodakis, N., Paragios, N., Tziritas, G.: MRF energy minimization and beyond via dual decomposition. IEEE Trans. PAMI 33 (2011) 531-552

18. Sontag, D., Globerson, A., Jaakkola, T.: Introduction to dual decomposition for inference. In Sra, S., Nowozin, S., Wright, S.J., eds.: Optimization for Machine Learning. MIT Press (2011) 
19. Boyd, S., Parikh, N., Chu, E., Peleato, B., Eckstein, J.: Distributed optimization and statistical learning via the alternating direction method of multipliers. Foundations and Trends in Machine Learning 3 (2011) 1-122

20. Ladický, L., Russell, C., Kohli, P., Torr, P.H.S.: Associative hierarchical random fields. IEEE Transactions on Pattern Analysis and Machine Intelligence 99 (2013) 1

21. Shotton, J., Winn, J.M., Rother, C., Criminisi, A.: TextonBoost: Joint appearance, shape and context modeling for multi-class object recognition and segmentation. In: ECCV (1). (2006) 1-15

22. Komodakis, N.: Efficient training for pairwise or higher order CRFs via dual decomposition. In: CVPR. (2011) 1841-1848 